\title{
Dartmouth Regimen
}

National Cancer Institute

\section{Source}

National Cancer Institute. Dartmouth Regimen. NCI Thesaurus. Code C63781.

A regimen consisting of tamoxifen, cisplatin, dacarbazine and carmustine used for the treatment of advanced-stage or metastatic melanoma. 